\title{
RESEARCH
}

Open Access

\section{Reproducibility of EEG functional connectivity in Alzheimer's disease}

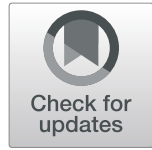

\author{
Casper T. Briels ${ }^{1,2^{*}}\left(\mathbb{D}\right.$, Deborah N. Schoonhoven ${ }^{1,2}$, Cornelis J. Stam² ${ }^{2}$, Hanneke de Waal ${ }^{1}$, Philip Scheltens ${ }^{1}$ and
} Alida A. Gouw ${ }^{2}$

\begin{abstract}
Background: Although numerous electroencephalogram (EEG) studies have described differences in functional connectivity in Alzheimer's disease (AD) compared to healthy subjects, there is no general consensus on the methodology of estimating functional connectivity in AD. Inconsistent results are reported due to multiple methodological factors such as diagnostic criteria, small sample sizes and the use of functional connectivity measures sensitive to volume conduction. We aimed to investigate the reproducibility of the disease-associated effects described by commonly used functional connectivity measures with respect to the amyloid, tau and neurodegeneration $(\mathrm{A} / \mathrm{T} / \mathrm{N})$ criteria.
\end{abstract}

Methods: Eyes-closed task-free 21-channel EEG was used from patients with probable AD and subjective cognitive decline $(S C D)$, to form two cohorts. Artefact-free epochs were visually selected and several functional connectivity measures (AEC(-c), coherence, imaginary coherence, PLV, PLI, WPLI) were estimated in five frequency bands. Functional connectivity was compared between diagnoses using AN(C)OVA models correcting for sex, age and, additionally, relative power of the frequency band. Another model predicted the Mini-Mental State Exam (MMSE) score of AD patients by functional connectivity estimates. The analysis was repeated in a subpopulation fulfilling the A/T/N criteria, after correction for influencing factors. The analyses were repeated in the second cohort.

Results: Two large cohorts were formed (SCD/AD; $n=197 / 214$ and $n=202 / 196)$. Reproducible effects were found for the AEC-c in the alpha and beta frequency bands $\left(p=6.20 \times 10^{-7}\right.$, Cohen's $d=-0.53 ; p=5.78 \times 10^{-4}, d=-$ $0.37)$ and PLI and $\mathrm{WPLI}$ in the theta band $\left(p=3.81 \times 10^{-8}, d=0.59 ; p=1.62 \times 10^{-8}, d=0.60\right.$, respectively). Only effects of the AEC-c remained significant after statistical correction for the relative power of the selected bandwidth. In addition, alpha band AEC-c correlated with disease severity represented by MMSE score.

Conclusion: The choice of functional connectivity measure and frequency band can have a large impact on the outcome of EEG studies in AD. Our results indicate that in the alpha and beta frequency bands, the effects measured by the AEC-c are reproducible and the most valid in terms of influencing factors, correlation with disease severity and preferable properties such as correction for volume conduction. Phase-based measures with correction for volume conduction, such as the PLI, showed reproducible effects in the theta frequency band.

Keywords: Alzheimer's disease, EEG, Functional connectivity, Reproducibility

\footnotetext{
*Correspondence: c.briels@amsterdamumc.nl

${ }^{1}$ Alzheimer Center Amsterdam, Department of Neurology, Amsterdam

Neuroscience, Vrije Universiteit Amsterdam, Amsterdam UMC, Amsterdam, the Netherlands

${ }^{2}$ Department of Clinical Neurophysiology, Amsterdam Neuroscience, Vrije Universiteit Amsterdam, Amsterdam UMC, Amsterdam, the Netherlands
}

(C) The Author(s). 2020 Open Access This article is licensed under a Creative Commons Attribution 4.0 International License, which permits use, sharing, adaptation, distribution and reproduction in any medium or format, as long as you give appropriate credit to the original author(s) and the source, provide a link to the Creative Commons licence, and indicate if changes were made. The images or other third party material in this article are included in the article's Creative Commons licence, unless indicated otherwise in a credit line to the material. If material is not included in the article's Creative Commons licence and your intended use is not permitted by statutory regulation or exceeds the permitted use, you will need to obtain permission directly from the copyright holder. To view a copy of this licence, visit http://creativecommons.org/licenses/by/4.0/ The Creative Commons Public Domain Dedication waiver (http://creativecommons.org/publicdomain/zero/1.0/) applies to the data made available in this article, unless otherwise stated in a credit line to the data. 


\section{Introduction}

Alzheimer's disease (AD) is the most common cause of dementia, and it is expected that 131.5 million people will be living with dementia in 2050 [1]. Electroencephalography (EEG) has been used to understand underlying mechanisms and support the diagnosis of $\operatorname{AD}[2,3]$, but also to observe treatment effects [4]. EEG is a relatively easy, cheap and widely available method and provides functional data with a high temporal resolution which makes it an interesting modality to investigate the disease.

Quantitative resting-state EEG data analyses can roughly be divided into three modalities: spectral decomposition, functional connectivity and network-based analyses. Each of these modalities has shown profound changes in $\mathrm{AD}$ compared to healthy subjects $[2,5,6]$. Spectral changes in AD are characterized by a general slowing of the dominant oscillatory brain rhythms. Typically, there is an increase in relative theta and delta power and a decrease in relative alpha and beta power $[2,7]$. The analyses of spectral changes are very straightforward and require no more than, for example, a fast Fourier transformation [8] of the oscillatory data. As a result, there is a broad consensus concerning the typical spectral changes in AD. Functional connectivity and network analyses on the other hand require more complex calculations and are subject to choices in the use of different properties of the EEG signal [9]. A broad and general consensus about changes in functional connectivity or brain networks is therefore more complicated.

Various studies have reported changes in functional connectivity in $\mathrm{AD}$. In general, $\mathrm{AD}$ is considered to be a disconnecting syndrome $[10,11]$, showing a lower functional connectivity in patients with dementia due to $\mathrm{AD}$ compared to healthy controls [3]. This conclusion is, however, not based on consistent methodology. Many studies suffered from small study populations and poor definition of study populations without using clinical [12] or research criteria [13]. But most importantly, although recommendations have been published $[9,14$, $15]$, there is an absence of a consensus on the methodology of estimating functional connectivity. It is, therefore, not surprising that different and sometimes conflicting results have been reported between measures of functional connectivity in AD. Reproducibility issues like these have recently gained more attention throughout the different fields of science [16]. As scientists are more eager to present new results or discoveries, there is limited motivation to reproduce and publish previously reported findings. Previous results could have been influenced by low statistical power, 'P-hacking' or publication bias, and it has been estimated that $85 \%$ of biomedical research efforts are not reproducible [17]. This methodological problem is often referred to as the 'reproducibility crisis'. A lack of reproducible results can impair the use of these measures in future (clinical) studies and even harm the credibility of the functional connectivity research field.

Examples of inconsistencies in functional connectivity literature, among others, can be found in the results reported for coherence. It is reported to be decreased in the alpha $(8-13 \mathrm{~Hz})$ band in $\mathrm{AD}$ subjects by multiple studies but some other studies have additionally reported an increase in delta $(0.5-4 \mathrm{~Hz})$ band coherence $[18,19]$ and an increase in theta $(4-8 \mathrm{~Hz})$ band coherence [20]. In another comparison of AD and cognitively normal patients, both the phase lag index (PLI) and imaginary coherence showed a decrease in functional connectivity in the beta $(13-30 \mathrm{~Hz})$ frequency band [21], where other studies showed differences in the alpha band PLI [22] and alpha [23] but also delta and theta band [24] imaginary coherence.

Some of the conflicting reports of change in functional connectivity may be explained by issues in study design such as differences in populations, sample size and (pre)processing. In addition, the choice of functional connectivity measure could also have had an influence on the results [25]. This could be due to differences in the use of EEG properties (i.e. amplitude, phase, frequency), the chosen bandwidth and regional changes. As shown by research in other neurodegenerative diseases, different functional connectivity measures can show different regional increases or decreases in the same or different bandwidths [26, 27]. Neurodegeneration also proves to be a nonlinear process which can cause different states in different stages of the disease $[28,29]$. As a result, it can be a valid procedure to look at different functional connectivity measures in different frequency bands simultaneously. However, inconsistencies can also occur due to a low reliability of the measure of functional connectivity. Large differences in test-retest reliability between measures have been reported [30, 31]. Moreover, even though a measure can be reliable, it also needs to be a valid measure of functional connectivity. An important factor influencing the validity of functional connectivity measures is the susceptibility to volume conduction [32]. Volume conduction can inflate or deflate the presented results [33], and therefore, functional connectivity measures without correction for this phenomenon are not valid measures of connectivity. This implies that the choice of functional connectivity measure can have an influence on the reproducibility, generalizability and validity of the results.

An influencing factor, briefly mentioned above, is that the diagnostic process has evolved from a mostly clinical evaluation to a biomarker-based process. Due to the presence of many comorbidities and clinical mimics of $\mathrm{AD}$, results from studies that involved patients without 
biomarker support might have been negatively influenced. Therefore, the AD research field has adopted a new research framework [13] implementing amyloid, tau and neurodegeneration $(\mathrm{A} / \mathrm{T} / \mathrm{N})$ criteria to ensure the results of current research are residues of the same biological process. In summary, it is important to evaluate the observed effects of $\mathrm{AD}$ on functional connectivity in light of the latest research framework, previously found discrepancies between test results and test-retest reliability issues of some functional connectivity measures.

This problem leads to the following question and aim of this study: which functional connectivity measures observe reproducible and valid EEG changes in $\mathrm{AD}$ and in which frequency bands do these changes occur? We aimed to answer these questions by designing a study in which we compared commonly used functional connectivity measures with respect to reproducibility, undesirable influence of covariates and correlation with disease severity. Data was gathered from a memory clinic to create a large population size with available EEG recordings to counter the statistical power limitations of earlier studies. The $\mathrm{A} / \mathrm{T} / \mathrm{N}$ criteria [13] were used to ensure AD-specific changes were observed. And in addition, a large second cohort was created to assess reproducibility. Other possible factors of influence, such as medication or other neurological and psychiatric disorders, were investigated. To our knowledge, no previous studies with EEG functional connectivity have been performed having such a large population size and availability of $\mathrm{A} / \mathrm{T} / \mathrm{N}$ classification.

To further evaluate the capacity of the functional connectivity measures to distinguish between different levels of disease severity, correlations with the Mini-Mental State Exam (MMSE) were estimated. Furthermore, collinearity between relative power and functional connectivity, to assess the potential influence, and the collinearity between the different functional connectivity measures were estimated. Although measures might be mathematically different, we expected that the observed variance could still be very similar between some of the measures due to shared ground principles.

\section{Methods}

\section{Clinical sample}

A test and a validation cohort were formed using clinical data from the clinical Amsterdam dementia cohort (ADC) in the Alzheimer Center VUmc, Amsterdam UMC, in the Netherlands [34]. All subjects visited the clinic as part of their diagnostic process during the period of 2002 to 2015 and were asked for informed consent to store their clinical data. EEGs were made as part of this process next to clinical assessment, neuropsychological test batteries, magnetic resonance imaging (MRI) or computed tomography scan (CT scan) and, when possible, assessment of amyloid burden by cerebrospinal fluid (CSF) or positron emission tomography (PET). Based on this gathered information, the final diagnosis was made by a multidisciplinary team of a neurologist, psychiatrist, neuropsychologist and radiologist during a consensus meeting according to the most recent clinical criteria. Data from the EEG recordings was used for various research purposes over time, and subjects were included based on the availability of epochs from these studies. Subjects with the clinical diagnosis of probable AD dementia [12] and subjective cognitive decline (SCD) with available EEG recordings were included.

\section{Cohort characteristics}

For this study, when biomarkers were available, subjects were classified using the $\mathrm{A} / \mathrm{T} / \mathrm{N}$ framework [13] by using CSF amyloid beta $(\mathrm{A} \beta)$ 1-42, p-tau and medial temporal atrophy (MTA [35], scored from 0 to 4 which resembles no to most severe atrophy) averaged over left and right. Drift-corrected $A \beta 1-42$ values were used with a cut-off of $813 \mathrm{pg} / \mathrm{mL}$ [36]. This correction was applied due to the gradual increase of Innotest A $\beta$ 1-42 CSF values over two decades of testing in the ADC, which could cause misclassification of amyloid status. When both amyloid-PET and CSF were available, the amyloid-PET was decisive. Cut-off for $\mathrm{p}$-tau was set at $52 \mathrm{pg} / \mathrm{mL}$, and the neurodegeneration cut-off was set at MTA $\geq 1$ based on visual assessment of T1-weighted acquisition MRI images [37]. Furthermore, patients were categorized by Fazekas score $(0-1$ and $\geq 2)$ based on visual assessment of fluid-attenuated inversion recovery (FLAIR) images on MRI [38].

The medical history of every patient was analysed and categorized with special interest for 3 possible confounding characteristics: vascular disease, central nervous system (CNS) disease and psychiatric disease. Vascular disease included only symptomatic vascular disease such as myocardial infarction and stroke. CNS disease included any major neurologic disease but also a medical history with significant brain injury. Psychiatric disease consisted of any major active or chronic psychiatric disease. Additionally, data of the use of any medication with potential effects on the EEG (acetylcholinesterase inhibitors, benzodiazepines, anti-epileptic drugs and antidepressants) was collected.

To create a cross-sectional design with internal validation, clinically diagnosed $\mathrm{AD}$ and $\mathrm{SCD}$ subjects were randomly allocated to two cohorts. Two subset populations of cohort 1 were additionally created based on the $\mathrm{A} / \mathrm{T} / \mathrm{N}$ framework and potential interfering covariates. Subpopulation 1: SCD subjects without amyloid (CSF or PET) and tau (p-tau) pathology versus AD subjects with amyloid and tau pathology. Subpopulation 2: SCD 
subjects without amyloid pathology, tau pathology, neurodegeneration $(\mathrm{MTA}<1)$, small vessel disease (SVD) (Fazekas $<2$ ) or use of interfering medication versus AD subjects with amyloid pathology, tau pathology, neurodegeneration $($ MTA $\geq 1)$ and without SVD (Fazekas $<2$ ) or use of interfering medication.

\section{EEG recordings}

Twenty minutes of eyes-closed task-free EEG recordings was made in a standardized protocol using the 21 electrode positions of the 10-20 system. Patients sat with eyes closed in a slightly reclined chair in a soundattenuated room. EEG technicians were alert on keeping the participants awake and to minimize artefacts. Acoustic stimuli were used when slow horizontal eye movements or slowing of the posterior alpha rhythm appeared. The sample frequency was set to $500 \mathrm{~Hz}$; electrode impedance was kept below $5 \mathrm{k} \Omega$ with low pass filter $\leq 70 \mathrm{~Hz}$, high pass filter $\geq 0.5 \mathrm{~Hz}$, and no notch filter; and the average reference was used. Trained researchers visually inspected the recordings looking for artefacts and the state of alertness. Four to five artefact-free epochs of $8.192 \mathrm{~s}$ were visually selected for each patient. The process of the visual selection process was previously described by Gouw and colleagues [39]. In short, the visual inspection of epochs was performed by a trained EEG researcher, based on the presence of a minimum of artefacts (e.g. excessive muscle activity, eye blinks) and drowsiness. If no sufficient quality was reached, the epochs were replaced by other epochs or the EEG was excluded from analyses when insufficient epochs could be included for analyses.

\section{EEG analyses}

The freely available 'Brainwave' software [40] was used to estimate functional connectivity. Mean global coherence (Coh), imaginary coherence (iCoh), phase locking value (PLV), amplitude envelope correlation (AEC), AEC with leakage correction (AEC-c), phase lag index (PLI) and weighted PLI (wPLI) were estimated in five frequency bands: broadband $(0.5-30$ $\mathrm{Hz})$, delta $(0.5-4 \mathrm{~Hz})$, theta $(4-8 \mathrm{~Hz})$, alpha $(8-13$ $\mathrm{Hz})$ and beta $(13-30 \mathrm{~Hz})$. Functional connectivity per electrode was estimated by averaging the values for each possible electrode pair per electrode (for example, the value of electrode Fp1 is the average of each potential electrode pair with electrode Fp1). The results of all electrodes were averaged to create global values. Fast Fourier transformation was used to estimate mean global relative delta, theta, alpha and beta power. The results of the different epochs were averaged for each subject.

\section{Functional connectivity measures}

Functional connectivity measures were chosen based on their usage in AD EEG literature [2, 5, 41, 42] and testretest reliability information [31]. The mathematical procedures to estimate these measures are described in the following paragraph in which we distinguish between measures with or without correction for volume conduction. Measures without correction are prone to signal leakage from channel to channel with the potential to deflate or inflate the connectivity estimate [33].

\section{Measures without correction for volume conduction}

Functional connectivity assesses functional communication between brain areas by estimating the level of synchronization of the EEG signals. To analyse statistical interdependencies, the wave-like EEG signal can be decomposed into different properties such as the frequency, amplitude or phase of the signal. The analytical signal $z(t)$ can be described as shown in Eq. 1 where $x(t)$ describes the real component of the time series, $\tilde{x}(t)$ the corresponding Hilbert transform, $A(t)$ the instantaneous amplitude and $\phi(t)$ the instantaneous phase.

$$
z(t)=x(t)+i \tilde{x}(t)=A(t) e^{i \phi(t)}
$$

The instantaneous amplitude (or amplitude envelope) and phase can be obtained from $z(t)$ by using, respectively, Eqs. 2 and 3.

$$
\begin{aligned}
& A(t)=\sqrt{[x(t)]^{2}+[\tilde{x}(t)]^{2}} \\
& \phi(t)=\arctan \frac{\tilde{x}(t)}{x(t)}
\end{aligned}
$$

How to obtain the Hilbert transform $\tilde{x}(t)$ of $x(t)$ is shown in Eq. 4, where PV refers to the Cauchy principal value.

$$
\tilde{x}(t)=\frac{1}{\pi} \mathrm{PV} \int_{-x}^{\infty} \frac{x(\tau)}{t-\tau} d \tau
$$

Coherence (Coh) [32, 43] is a functional connectivity measure which analyses synchronization in the frequency domain. Coherence is the absolute value of Coherency $c$ which can be calculated (after applying the Hilbert transformation) according to Eq. 1 in Table 1. In this equation, $A$ represents the instantaneous amplitude of signal 1 or 2 and $\Delta \phi$ the instantaneous phase difference between the two signals.

The stability of the phase difference between two time series can be estimated by using Eq. 2 in Table 1 and in literature by it has been termed as the phase locking value (PLV) [44] or the phase coherence [45]. Where the phase coherence uses the Hilbert transform prior to Eq. 2 in Table 1, the PLV uses a wavelet-based analysis. 
Table 1 The equations of the used functional connectivity measures. For each functional connectivity measure, it is indicated whether they observe associations between signals based on amplitude $(A)$, phase $(P)$ or both $(A / P)$

\begin{tabular}{|c|c|c|}
\hline Measure & & Equation \\
\hline 1. Coherency & $\begin{array}{l}\text { A/ } \\
P\end{array}$ & $c=\frac{\left\langle A_{1} A_{2} e^{i\langle\varphi}\right\rangle}{\sqrt{\left\langle A_{1}^{2}\right\rangle\left\langle A_{2}^{2}\right\rangle}}$ \\
\hline $\begin{array}{l}\text { 2. Phase locking value/phase } \\
\text { coherence }\end{array}$ & $P$ & $R=\left|\left\langle e^{i \Delta \varphi_{i j}(t)}\right\rangle\right|=\left|\frac{1}{N} \sum_{k=0}^{N-1} e^{i \Delta \varphi\left(t_{k}\right)}\right|$ \\
\hline 3. Amplitude envelope & A & $A(t)=\sqrt{[x(t)]^{2}+[\tilde{x}(t)]^{2}}$ \\
\hline 4. Imaginary coherence & & $\operatorname{Im}\{C\}=\frac{\left\langle A_{1} A_{2} \sin \Delta \varphi\right\rangle}{\sqrt{\left\langle A_{1}^{2}\right\rangle\left\langle A_{2}^{2}\right\rangle}}$ \\
\hline 5. Phase lag index & $P$ & $\mathrm{PLI}=\left|\left\langle\operatorname{sign}\left[\sin \left(\Delta \varphi\left(t_{k}\right)\right)\right]\right\rangle\right|$ \\
\hline 6. Weighted phase lag index & P & $\mathrm{WPLI} \equiv \frac{|E\{\mathfrak{I}(Z)\}|}{E\{\mathfrak{I}(Z)\}}=\frac{|E\{|\mathfrak{I}(Z)| \operatorname{sign}(\mathfrak{I}(Z))\}|}{E\{|\mathfrak{I}(Z)|\}}$ \\
\hline
\end{tabular}

Literature has, however, shown that both approaches produce similar results [46].

The amplitude envelope correlation (AEC) [47] is an amplitude-based measure which estimates the Pearson correlation between the envelopes of the amplitudes of time series (Eq. 3 in Table 1). The amplitude envelopes are calculated by using the Hilbert transform of the time series.

\section{Measures with correction for volume conduction}

Several methods have been developed to correct for volume conduction. The corrected amplitude envelope correlation (AEC-C) [48] uses pair-wise orthogonalization prior to the AEC calculations described in the 'Measures without correction for volume conduction' section. The averaged result of a pair-wise orthogonalization in both directions, $X$ to $Y$ and $Y$ to $X$, was used.

The imaginary coherence (iCoh) [49] can be estimated with the imaginary part of coherency according to Eq. 4 in Table 1. Where the imaginary coherence is also based on the amplitude of the signal, the phase lag index (PLI) [21] is a solely phase-based measure with correction for volume conduction. It estimates the asymmetry of the distribution of phase differences $\Delta \phi(t)$ between time series. Yielding low values for median phase differences of $0 \bmod \pi$. It can be estimated according to Eq. 5 in Table 1. Due to the discontinuity of the index, the PLI may be hindered by small perturbations, around a phase difference of $0 \bmod \pi$, which cause phase lags to turn into leads and vice versa. The weighted phase lag index (wPLI) [50] corrects for this phenomenon by using the magnitude of the imaginary component of the crossspectrum as a weight for the phase lags (Eq. 6 in Table 1 ) where $\Im(Z)$ is the imaginary component $(\sin \Delta \phi)$ of signal $Z$. Unfortunately, this measure depends upon both the consistency and the magnitude of the phase difference.

\section{Statistical analysis}

All statistical analyses were performed using SPSS statistics software (version 24.0.0.1). Available demographic and medical characteristics were described, and differences between SCD and AD subjects in both cohorts were assessed by independent $t$ test, chi-square test, Fisher's exact test or Mann-Whitney $U$ test where appropriate. Normality of distribution of the variables was checked by histograms and Q-Q plots.

Distribution of the functional connectivity measures was also checked by histograms and Q-Q plots, and when appropriate, variables were log transformed. Differences in functional connectivity measures between $\mathrm{AD}$ and SCD subjects were determined by two models of analysis of (co)variance (AN(C)OVA). Model 1 applied correction for the covariates age and sex. Model 2 corrected for age, sex and the relative power of the bandwidth in which the functional connectivity was measured. ANOVA on ranks [51] was performed for variables that could not be successfully log transformed. After each of the ANOVA models, the effect size was estimated by Cohen's $d$ [52]. In addition, various demographic and medical characteristics were separately added as a covariate to model 1 to check for any interfering effects. To observe regional reproducibility, we have averaged the individual channels into 4 regions: frontal (channels Fp1, Fp2, F3, F4, F7, F8, Fz), temporal (channel T3, T4, T5, T6), central (channel C3, C4, Cz) and parieto-occipital (P3, P4, Pz, O1, O2). ANCOVA model 1 (with correction for age and sex) was repeated for these regional values over each bandwidth in cohort 1 (including the 2 subpopulations) and cohort 2 .

False discovery rate (FDR) [53] correction was applied to $p$-values of the demographic and medical characteristic comparisons between the SCD and AD groups. FDR correction was furthermore applied to the $p$-values of the ANOVA models correcting for the multiple testing in different bandwidths of each functional connectivity measure. Due to the expected presence of high collinearity between the tested functional connectivity measures (see the 'Collinearity between functional connectivity measures' section), the number of hypotheses tested in the FDR correction was set to 5 (the number of tested bandwidths). This choice was also made due to the use of a validation cohort which will in turn also reduce the number of false positive results. $p$-values shown were FDR corrected, and a threshold of $p<0.05$ was maintained.

Correlations between global values of the different functional connectivity markers were explored, and the level of collinearity was assessed. In addition, correlations between functional connectivity and relative power 
were investigated. The correlations were investigated with Pearson and Spearman correlations where appropriate.

\section{Results}

\section{Demographic and medical cohort characteristics}

Two cohorts were created to assess cross-sectional differences in functional connectivity. Cohort 1 consisted of 197 SCD and 214 AD subjects, whereas cohort 2 consisted of 202 SCD and 196 AD subjects. Available characteristics of both cohorts are shown in Table 2. Most notable was the significant difference in age between SCD and AD subjects in both cohorts. SCD subjects were younger in both cohorts (mean difference $(\Delta$ mean) of 5 and 9 years in cohorts 1 and 2, respectively). No significant differences were found in medical history between SCD and AD subjects. Significant differences in MMSE, CSF, PET and MRI results were in concordance with the SCD and AD diagnoses. Across cohorts, SCD subjects had a slightly higher MMSE in cohort 2 ( $\triangle$ median $=1$ ). AD subjects had a slightly higher MMSE
$(\Delta$ median $=1)$ and MTA $(\Delta$ median $=0.5)$ and lower total tau $(\Delta$ mean $=4)$ in cohort 2.

Subpopulations were formed based on the availability of CSF and amyloid-PET biomarkers. In subpopulation 1 , all AD subjects with positive amyloid and tau biomarkers $(n=135 ; \mathrm{A}+\mathrm{T}+)$ and SCD subjects without evidence for $\mathrm{AD}$, thus with negative amyloid and tau biomarkers $(n=97 ; \mathrm{A}-\mathrm{T}-)$, were selected. Even more strict criteria were used additionally for subpopulation 2, excluding patients with potential interfering comedication or SVD and selection based on MTA score as a marker of neurodegeneration $(\mathrm{A}+\mathrm{T}+\mathrm{N}+$ for $\mathrm{AD}$; $\mathrm{A}$ $-\mathrm{T}-\mathrm{N}-$ for $\mathrm{SCD})$. This resulted into $71 \mathrm{SCD}$ and $41 \mathrm{AD}$ subjects from cohort 1 .

\section{Functional connectivity}

Differences in global functional connectivity between SCD and AD subjects in cohort 1 were estimated by ANOVA model 1. A summary of the observed effect sizes is shown in Fig. 1. A more detailed description, including mean value, standard deviation and $p$-value, of the significant effects shown in Fig. 1 can be found in

Table 2 Characteristics of subjective cognitive decline (SCD) and dementia due to Alzheimer's disease (AD) subjects in both cohorts. The count (n), mean or median with percentage (\%), standard deviation (SD) or interquartile range (IQR) are shown for each variable. MMSE Mini-Mental State Exam, CNS central nervous system, MTA Medial Temporal Atrophy score, A $1-42$ amyloid beta 1-42, $t$-tau total tau, $p$-tau phosphorylated tau

\begin{tabular}{|c|c|c|c|c|}
\hline \multirow[b]{2}{*}{ Characteristic } & \multicolumn{2}{|l|}{ Cohort 1} & \multicolumn{2}{|l|}{ Cohort 2} \\
\hline & $\begin{array}{l}S C D \\
n=197\end{array}$ & $\begin{array}{l}\mathrm{AD} \\
n=214\end{array}$ & $\begin{array}{l}S C D \\
n=202\end{array}$ & $\begin{array}{l}A D \\
n=196\end{array}$ \\
\hline Female $(n, \%)$ & $76(39 \%)$ & $104(49 \%)$ & 89 (44\%) & $94(48 \%)$ \\
\hline Age (mean, SD) & $62 \pm 8$ & $67 \pm 8^{\mathrm{a}}$ & $60 \pm 10$ & $69 \pm 10^{a}$ \\
\hline MMSE (median, IQR) & $28(27-29)$ & $21(17-24)^{a}$ & $29(28-30)^{\mathrm{b}}$ & $22(18-25)^{a, b}$ \\
\hline Vascular disease $(n, \%)$ & $34(17 \%)$ & $40(19 \%)$ & $35(17 \%)$ & $32(16 \%)$ \\
\hline CNS disease $(n, \%)$ & $20(10 \%)$ & $14(7 \%)$ & $22(11 \%)$ & $19(10 \%)$ \\
\hline Psychiatric disease ( $n, \%)$ & $6(3 \%)$ & $8(4 \%)$ & $5(2 \%)$ & $1(1 \%)$ \\
\hline Antidepressants $(n, \%)$ & $20(10 \%)$ & $26(12 \%)$ & $16(8 \%)$ & 19 (10\%) \\
\hline Benzodiazepines $(n, \%)$ & $11(6 \%)$ & $12(6 \%)$ & $18(9 \%)$ & $15(8 \%)$ \\
\hline Anti-epileptic drugs ( $n, \%)$ & $4(2 \%)$ & $3(1 \%)$ & $4(2 \%)$ & $3(2 \%)$ \\
\hline Acetylcholinesterase inhibitors $(n, \%)$ & $0(0 \%)$ & $16(7 \%)^{a}$ & $1(0 \%)$ & $18(9 \%)^{a}$ \\
\hline MRI & $n=176$ & $n=199$ & $n=175$ & $n=156$ \\
\hline MTA (median, IQR) & $0(0-0.5)$ & $1(0.5-2)^{\mathrm{a}}$ & $0(0-0.5)$ & $1.5(1-2.5)^{a, b}$ \\
\hline Fazekas (median, IQR) & $1(0-1)$ & $1(0-1)$ & $0(0-1)$ & $1(0.5-1)^{a}$ \\
\hline Cerebrospinal fluid & $n=183$ & $n=169$ & $n=120$ & $n=141$ \\
\hline$A \beta$ 1-42 (mean, SD) & $1031 \pm 268$ & $663 \pm 136^{a}$ & $1090 \pm 197$ & $694 \pm 162^{a}$ \\
\hline t-tau (mean, SD) & $302 \pm 214$ & $731 \pm 413^{a}$ & $267 \pm 119$ & $610 \pm 321^{a, b}$ \\
\hline p-tau (mean, SD) & $50 \pm 23$ & $89 \pm 35^{a}$ & $47 \pm 16$ & $85 \pm 38^{\mathrm{a}}$ \\
\hline Amyloid-PET & $n=32$ & $n=34$ & $n=11$ & $n=17$ \\
\hline Positive PET $(n, \%)$ & $12(38 \%)$ & $34(100 \%)^{a}$ & $3(27 \%)$ & $14(82 \%)^{a}$ \\
\hline
\end{tabular}

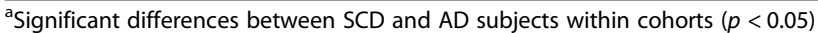

${ }^{\mathrm{b}}$ Significant differences in the same diagnostic group across cohorts (e.g. SCD versus SCD and AD versus AD) $(p<0.05)$ 
supplementary Table 1 . Figure 1 only shows the global effects that were both significant in cohort 1 , subpopulation 2 and validation cohort 2.

Clear differences between functional connectivity measures can be observed in Fig. 1. Firstly, the AEC-c, AEC, coherence and PLV showed lower functional connectivity in the alpha $(d=-0.5 ; d=-0.9 ; d=-0.7 ; d=-0.5$, respectively) and beta $(d=-0.4 ; d=-0.4 ; d=-0.3$; no effect of the PLV, respectively) frequency bands in $\mathrm{AD}$ subjects. Secondly, the imaginary coherence, PLI and wPLI observed higher functional connectivity in the theta frequency band in $\mathrm{AD}$ subjects $(d=0.5 ; d=0.6$; $d=0.6$, respectively). Thirdly, the delta frequency band and broadband functional connectivity did not show robust group differences. The largest difference between the AD and SCD subjects was observed in the uncorrected AEC in the alpha band (Cohen's $d=-0.90, p=$ $8.5 \times 10^{-18}$, model 1$)$. This effect remained the largest when the population was selected based on amyloid and phosphorylated tau (subpopulation $1, d=-0.83, p=$ $1.8 \times 10^{-9}$ ) or the complete $\mathrm{A} / \mathrm{T} / \mathrm{N}$ criteria, exclusion of SVD and potential interfering medication (subpopulation $\left.2, d=-1.03, p=4.6 \times 10^{-7}\right)$. In cohort 2 , this difference was validated to be the largest of the tested measures $\left(d=-0.68, p=6.2 \times 10^{-11}\right)$.

The significant effects observed in cohort 1 , cohort 2 and both subpopulations are shown in Fig. 2. Although minor differences in observed effects and effect sizes are present, most effects observed in the entire cohort 1 are generally similar to the effects in the subpopulations and the validation cohort. Notable differences across cohorts were a reproducible effect on the delta band AEC-c in cohorts 1 and 2 and subpopulation $1(\mathrm{~A} / \mathrm{T})$ but not $2(\mathrm{~A} / \mathrm{T} / \mathrm{N})$ (see topographic distributions below) and a reproducible effect of the alpha band PLI and wPLI in cohorts 1 and 2 but not in subpopulations 1 and 2 .

In order to look at the topographic distribution of the observed effects, the AEC-c and PLI were specifically selected because these measures correct for volume conduction and showed reproducible global effects. The topographic distributions of AEC-c and PLI in cohort 1 are shown in supplementary Figure 1. The reproducibility of the regional effects on the AEC-c and PLI is shown in supplementary Figure 2A and $2 \mathrm{~B}$. For all regions, a reproducible increase in theta band PLI and a decrease in alpha band AEC-C were found. The effect of the PLI was the strongest in the temporal channels $\left(d=0.66, p=9.2 \times 10^{-11}\right)$, and the effect of the AEC-c was the strongest in the temporal $\left(d=-0.56, p=3.2 \times 10^{-8}\right)$ and parietooccipital $\left(d=-0.56, p=2.9 \times 10^{-8}\right)$ channels. Furthermore, the AEC-c showed reproducible effects in the delta band AEC-c of the central channels and beta band AEC-c of the frontal, temporal and parietooccipital channels.

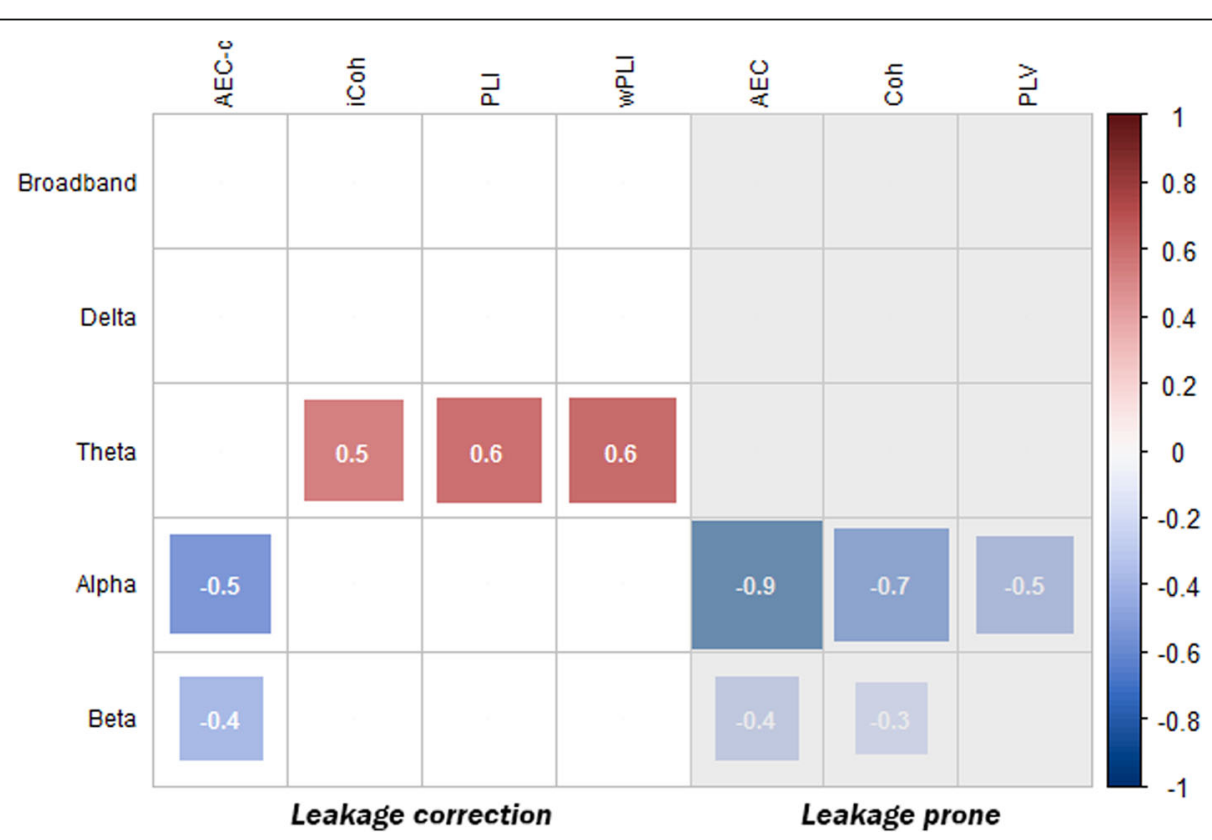

Fig. 1 Summary of observed differences in ANOVA model 1, shown as effect size (Cohen's d), between AD and SCD subjects for each of the functional connectivity measures and bandwidths. The significant effect sizes of the comparisons made in the entire cohort $1(n=411)$ are shown. Effects that could not be reproduced in the subset populations or cohort 2 were left out. Red blocks represent a higher and blue blocks a lower level of functional connectivity in AD subjects compared to SCD subjects. The size of the blocks and the number shown in the blocks represent the size of the effect. Results of functional measures susceptible to signal leakage are shaded in grey 


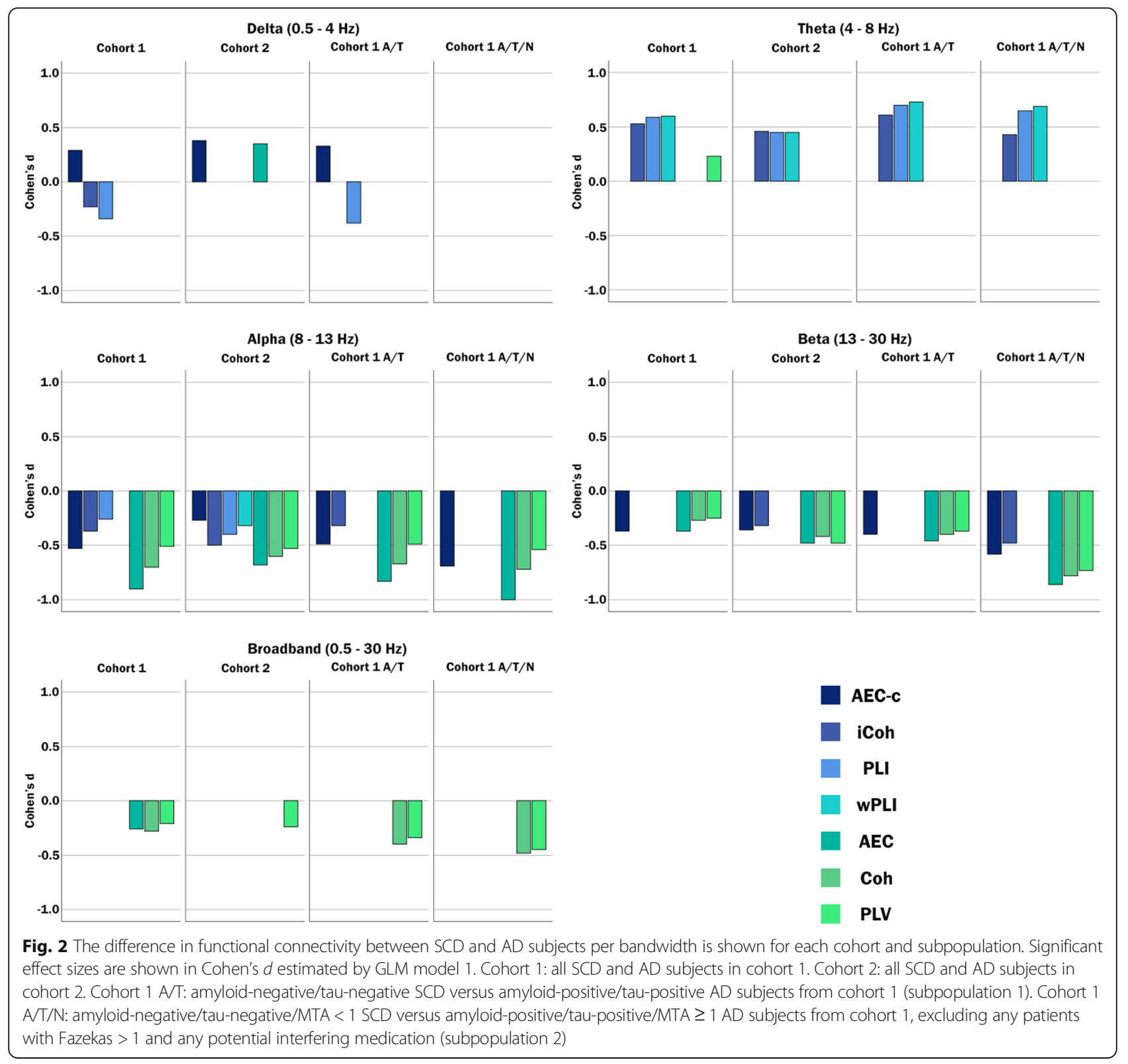

\section{Influence of medication and medical history}

The influence of potential covariates (medication: acetylcholinesterase inhibitors, benzodiazepines, anti-epileptic drugs, antidepressants; medical history: CNS disease, psychiatric disease, presence of SVD) was investigated by adding the variables to model 1 . The only significantly observed (small) effects were a decrease in beta band PLI, wPLI and PLV $(d=-0.22, p=0.027 ; d=-0.22, p=0.028$; $d=-0.24, p=0.020)$ and an increase in delta band wPLI $(d=-0.22, p=0.031)$ associated with antidepressants. Furthermore, a decrease in beta band PLV $(d=-0.24, \mathrm{p}=$ 0.020 ) was associated with the presence of SVD. Adding these covariates to model 1 did not change the observed effects between $\mathrm{AD}$ and $\mathrm{SCD}$ subjects.

\section{Effect of relative power}

To evaluate the relation of relative power with different functional connectivity measures, Pearson and Spearman correlations were estimated between these variables. The correlations were calculated between the functional connectivity in each frequency band and the relative power in that frequency band (e.g. correlation between alpha band AEC-c and relative alpha power). The results can be found in Fig. 3.

Various functional connectivity measures showed very high correlations with the relative power in the corresponding bandwidth. These effects were most pronounced in the theta and alpha bands. The two highest correlations were between the alpha band coherence and 


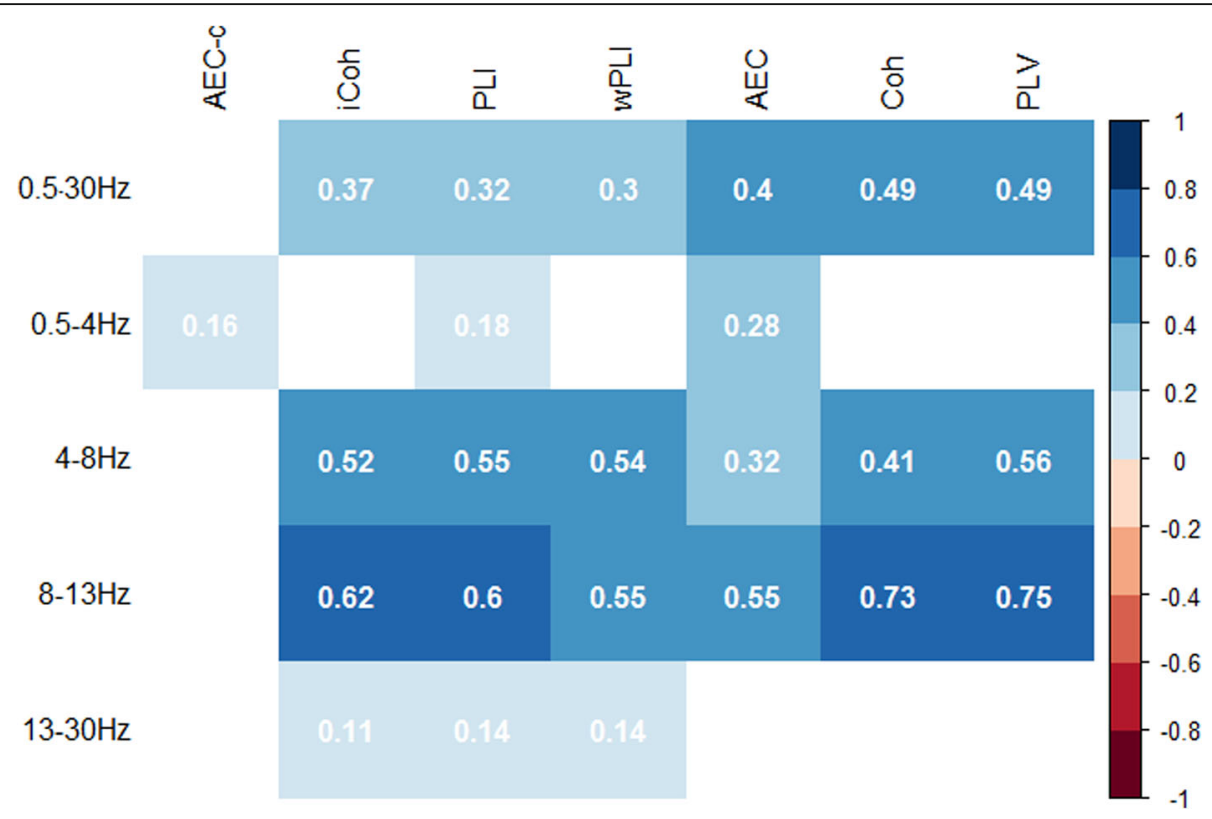

Fig. 3 Correlation of different functional connectivity measures with the relative power in the corresponding frequency band. The functional connectivity measures are shown on the $x$-axis and the band power on the $y$-axis. Only significant correlations are shown, indicated by the correlation coefficient $(r)$ which is also indicated by the colour gradient from $r=-1$ (dark red) to $r=+1$ (dark blue)

PLV with the relative alpha power $(r=0.73, p=$ $4.4 \times 10^{-67} ; r=0.75, p=4.0 \times 10^{-72}$, respectively). In contrast to the other functional connectivity measures and apart from a small effect $(r=0.16, p=0.001)$ in the delta frequency band, the AEC-c was not correlated to the relative power.

To observe how much of the variance of the effects between $\mathrm{AD}$ and SCD subjects observed in ANOVA model 1 could be explained by changes in relative power, the analyses were repeated with additional correction for relative band power (model 2). In line with the analysis of model 1 , shown in Fig. 1, the results of model 2 are shown in Fig. 4. Again, only the effects of cohort 1, which were significant in cohort 1 , subpopulation 2 and validation cohort 2, are shown. More detailed results of Fig. 4 can be found in supplementary Table 2.

In comparison with ANOVA model 1, the effects observed by the AEC-c remained stable in model 2 (model 1: alpha band $d=-0.53, p=1.24 \times 10^{-7}$; beta band $d=$ $-0.37, p=2.31 \times 10^{-4}$; model 2 : alpha band $d=-0.57$, $p=1.9 \times 10^{-8}$; beta band $d=-0.42, p=3.3 \times 10^{-5}$ ). The AEC effect also remained stable in the beta band but decreased in the alpha band compared to model 1. The observed effects of the coherence (alpha and beta bands) and PLV (alpha band) in model 1 disappeared in model 2. The theta band effects of the imaginary coherence, PLI and weighted PLI disappeared as well. In contrast, where no theta band effect was found with the coherence and PLV in model 1, these measures showed a reduced theta band functional connectivity in $\mathrm{AD}$ subjects, with the largest effect for the PLV $(d=-0.62, p=$ $\left.1.0 \times 10^{-9}\right)$, in model 2 .

\section{Disease severity and functional connectivity}

In Fig. 1, the functional connectivity measures were shown that show consistent differences between SCD and $\mathrm{AD}$. However, a functional connectivity measure which is indicative for disease severity would be even more valid and clinically relevant. Therefore, Pearson correlation coefficients were calculated between the valid connectivity measures in Fig. 1 and disease severity represented by the MMSE score (Table 3). Of the 12 observed functional connectivity measures from model 1 , only 4 showed correlations with disease severity in the AD subjects, with the largest, but modest, effects of the AEC-c $(r=0.14, p=0.0054)$ in the alpha band and the $\operatorname{AEC}(r=0.13, p=0.0073)$ in the beta band.

\section{Collinearity between functional connectivity measures}

Some of the effects observed by functional connectivity markers in ANOVA model 1 were very similar in size. To observe whether they measured similar differences between subjects, Pearson correlations between all functional connectivity measures were estimated. In addition, due to the high correlations with relative power observed in the 'Effect of relative power' section, the analyses were repeated with statistical correction for relative 


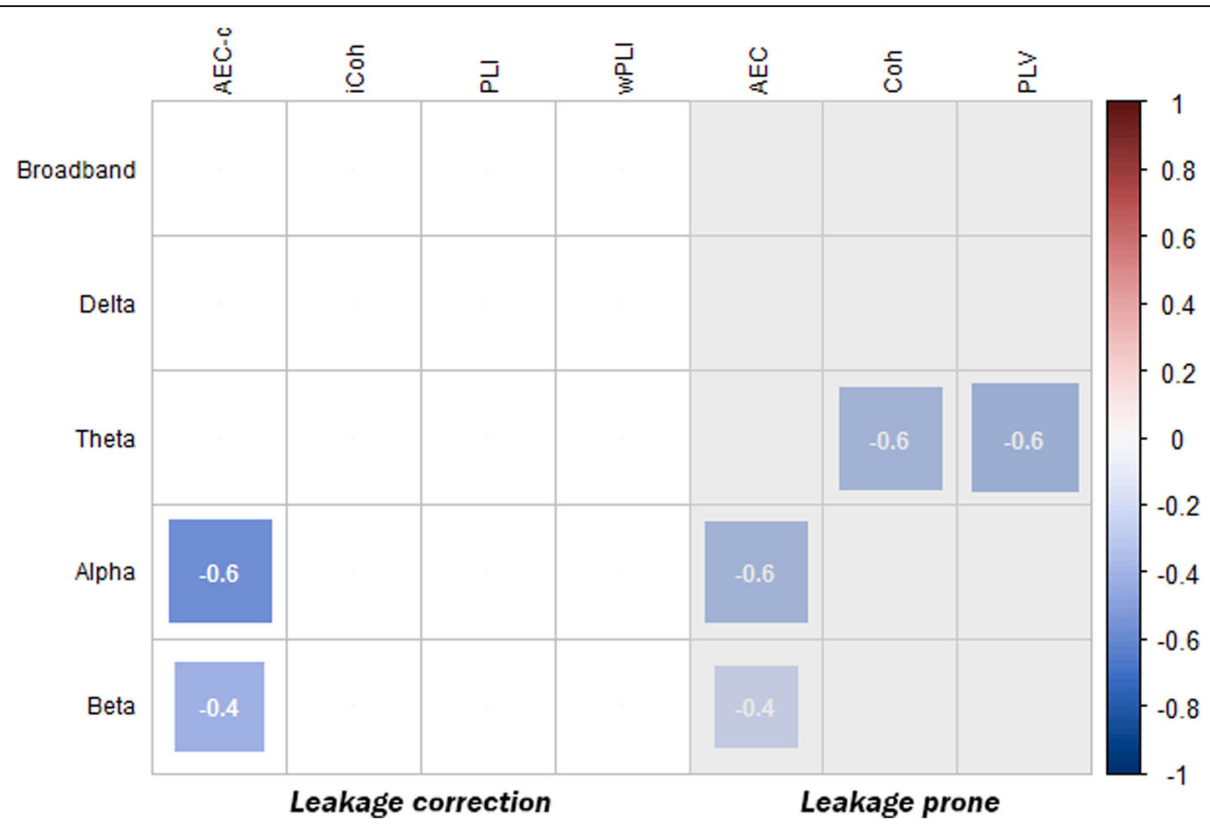

Fig. 4 Summary of observed differences in ANOVA model 2, shown as effect size (Cohen's d), between AD and SCD subjects for each of the functional connectivity measures and bandwidth. The significant effect sizes of the comparisons made in the entire cohort $1(n=411)$ are shown. Effects that could not be reproduced in the subset populations or cohort 2 were left out. Red blocks represent a higher and blue blocks a lower level of functional connectivity in AD subjects compared to SCD subjects. The size of the blocks and the number shown in the blocks represent the size of the effect. Results of functional measures susceptible to signal leakage are shaded in grey

Table 3 Pearson correlation coefficients ( $r$ ) between functional connectivity (FC) and disease severity represented by MMSE. Correlations were estimated for the entire population (cohorts 1 and 2) and the AD subjects only. Bold $r$ values indicate a significant $(p<0.05)$ correlation

\begin{tabular}{llll}
\hline FC \\
$\begin{array}{l}\text { measure } \\
\text { versus }\end{array}$ & All subjects $(\boldsymbol{n}=809)$ & & AD only $(\boldsymbol{n}=410)$ \\
\cline { 2 - 3 } & $\boldsymbol{r}$ & & $\boldsymbol{r}$-value
\end{tabular}

MMSE

\begin{tabular}{|c|c|c|c|c|}
\hline \multicolumn{5}{|c|}{ Theta $(4-8 \mathrm{~Hz})$} \\
\hline iCoh & -0.13 & $1.41 \times 10^{-4}$ & -0.06 & 0.23 \\
\hline PLI & -0.13 & $2.79 \times 10^{-4}$ & -0.06 & 0.23 \\
\hline WPLI & -0.13 & $1.71 \times 10^{-4}$ & -0.06 & 0.21 \\
\hline \multicolumn{5}{|c|}{ Alpha $(8-13 \mathrm{~Hz})$} \\
\hline$A E C-C$ & 0.21 & $3.2 \times 10^{-9}$ & 0.14 & 0.0054 \\
\hline $\mathrm{AEC}$ & 0.35 & $6.1 \times 10^{-25}$ & 0.14 & 0.0065 \\
\hline Coh & 0.27 & $6.4 \times 10^{-15}$ & 0.04 & 0.40 \\
\hline PLV & 0.23 & $7.3 \times 10^{-11}$ & 0.08 & 0.13 \\
\hline \multicolumn{5}{|c|}{ Beta $(13-30 \mathrm{~Hz})$} \\
\hline AEC-C & 0.21 & $1.8 \times 10^{-9}$ & 0.08 & 0.12 \\
\hline AEC & 0.26 & $1.0 \times 10^{-13}$ & 0.13 & 0.0073 \\
\hline Coh & 0.22 & $3.1 \times 10^{-10}$ & 0.12 & 0.015 \\
\hline
\end{tabular}

power. Results of the theta, alpha and beta bandwidths can be found in supplementary Figure 3A-C. These figures show similar clusters of correlating functional connectivity markers in the different bandwidths. High correlations between the imaginary coherence, PLI, wPLI and PLV are evident. In contrast, the AEC-c showed only strong correlations with its uncorrected version.

\section{Discussion}

\section{Main outcomes}

By using large well-characterized cohorts and the most recent NIA-AA research framework for Alzheimer's disease [13], we aimed to find the most reproducible and robust changes of EEG functional connectivity in Alzheimer's disease. The most reproducible and robust observed effects were decreases in the alpha and beta band AEC-c functional connectivity in the AD subjects. Furthermore, sensitivity of different functional connectivity measures appeared to be highly bandwidth specific, as most volume conduction-corrected phase-based measures showed reproducible increased functional connectivity in the theta band, whereas amplitude-based or more general connectivity measures showed decreased functional connectivity in the alpha and beta bands.

The conclusion that the decreases in alpha and beta band AEC-c were the most reproducible and robust functional connectivity estimates in $\mathrm{AD}$ is based upon the following observations. First, the AEC-c retained 
reproducible results in the test and validation cohorts and these effects were amplified when the analyses were repeated in a subpopulation of patients fulfilling the A/ $\mathrm{T} / \mathrm{N}$ criteria. This implies that these effects are (Alzheimer's) disease specific. Secondly, the observed effects were robust. The effects of the alpha and beta band AEC-c were not influenced by relative band power, several demographic variables, (co)morbidities or interfering medication. Thirdly, the AEC-c was not only a diseasespecific marker of AD but also correlated with disease severity. In AD subjects, lower alpha band AEC-c values modestly correlated with lower MMSE scores. Lastly, the AEC-c has been shown to be a reliable functional connectivity measure in previous test-retest reliability research [31]. Colclough and colleagues have tested all the functional connectivity measures used in our study on group-level repeatability and within-subject and between-subject consistency. Overall, the AEC-c showed the most consistent results compared to other measures corrected for volume conduction.

We propose the effects in the frequency bands and functional connectivity measures as shown in Fig. 1 as most reproducible. These effects remained present in large cohorts (i.e. high statistical power) but also after selection for $\mathrm{AD}$-specific biomarkers in a smaller subpopulation. It is however most preferable to use functional connectivity measures that are insensitive to volume conduction or change in relative power. When looking at the summarized effects of all functional connectivity measures between $\mathrm{SCD}$ and $\mathrm{AD}$, two major trends between different measures can be observed. Volume conduction-corrected phase-based measures (PLI, wPLI and iCoh) showed an increase in theta band functional connectivity, whereas amplitude or more mixed connectivity-based measures (AEC(-c), coherence, PLV) showed decreases in the alpha and beta bands. Therefore, future studies could consider using both a phasebased measure in the theta band and an amplitudebased measure in the alpha or beta band for their study or trial design. More research is needed to investigate the underlying processes of these distinctly different effects in phase and amplitude connectivity in AD. Phaseand amplitude-based functional connectivity measures could potentially capture different aspects of different patho- or neurophysiological processes. A review by Engel and colleagues proposed that amplitude- (or envelope) based connectivity has a close relation with the structural network and is relatively robust against state changes [54]. Phased-based connectivity appeared to be less related to the structural network and showed a stronger state dependence. On the other hand, recent literature has also shown that modulation of phaseamplitude coupling in long-range circuits may be highly relevant in cognitive functioning [55], indicating that amplitude and phase connectivity could be two interacting modalities. Changes in phase-based connectivity could affect amplitude-based connectivity (in the same or other regions) and vice versa.

\section{Main outcomes compared to literature}

We have compared our results with previous findings in literature. The decrease in alpha and beta band AEC-c is supported by previous MEG and EEG studies [42, 56]. Our regional analyses showed a widespread decrease of alpha and beta band AEC-c, with the exception of the beta band AEC-c in the central channels. In comparison, an EEG study by Nuñez and colleagues [56] compared the alpha and beta AEC-c in healthy controls with AD subjects and found a similar widespread decrease in alpha and beta band AEC-c. MEG has a higher spatial resolution than EEG, offering a potential explanation why in a MEG study between elderly controls and AD subjects, Koelewijn and colleagues found the most outspoken decreases in beta band AEC-c specifically in the bilateral (middle-) superior temporal and parietal cortex [42] instead of a general widespread decrease. The sensitivity of the AEC-c in the alpha and beta bandwidths might not be surprising because they are in line with an earlier methodological study by Hipp and colleagues [48] which has shown the AEC-c to be the most sensitive to changes in these bandwidths.

A decrease in alpha band coherence in $A D$ is widely known in the present literature $[2,41]$ and was reproduced in our study. A decrease or increase in delta and theta band coherence was reported by some articles [18-20] but could not be reproduced in either of our cohorts and subpopulations. Various other studies could also not reproduce these results $[2,41]$. Potential explanations include small sample sizes, poor inclusion criteria and suboptimal control populations.

A global and widespread increase in theta band phasebased measures with correction for volume conduction (PLI, wPLI, iCoh) is in line with some previous studies $[24,57]$; however, we could not reproduce a decrease in the alpha band $[5,23]$. We did observe some alpha band effects with the phase-based measures in the entire cohort but these effects disappeared when the NIA-AA research criteria [13] are applied and potential interfering factors are eliminated. The effects could therefore potentially be caused by common co-pathology such as vascular disease [58] or commonly missed diagnoses such as Lewy body dementia (DLB) [59]. This could influence the results as shown by a recent study by van der Zande and colleagues [60] where profound lower alpha band PLI values for DLB subjects compared to AD subjects were found. Another explanation could be the low testretest reliability of the phase-based functional connectivity measures [31]. 


\section{Effect of relative power}

Many of the tested functional connectivity measures had a high correlation with relative power in the corresponding frequency band. Only the AEC-c seems to be independent of relative power in most of the frequency bands. This was also represented in ANOVA model 2, where the effects observed by the AEC-c were not affected by a statistical correction to relative power. The high correlation of the AEC, coherence, PLV and, to lesser extent, the imaginary coherence with relative power is possibly due to the susceptibility of these measures to volume conduction effects $[32,61]$ and potentially due to changes in signal to noise ratio (SNR). An increase in relative power (and subsequently signal to noise ratio) is known to be correlated with an increase in volume conduction [32, 62]. Another consideration is that previous research has shown that there is a physiological relation between oscillatory activity and functional connectivity. Tewarie and colleagues have, for example, shown a relation between the amplitude of a signal and the dynamic functional connectivity measured by phase-difference derivative (PDD) [63]. This study showed that the PDD and the amplitude positively correlate in resting state MEG, sensorimotor task MEG and data based on a neuronal model. Nonetheless, it remains unclear what the exact relations are between oscillatory activity and the different functional connectivity measures applied in our study. The correlation of functional connectivity measures without correction for volume conduction with relative power was the strongest in the alpha frequency band, which implies that interpretation of results within this functional connectivity band should be made with caution. One of the caveats of applying statistical correction for relative power in cross-sectional studies is, however, potential overcorrection due to group effects. Due to the difference in relative power and functional connectivity between SCD and AD subjects, it is likely that these measures show similar relationships. Statistical correction for the strong effect of an increased global theta power in $\mathrm{AD}$, for example, may diminish the observed effect of volume conductioncorrected measures such as the PLI and wPLI in the theta band. Contrastingly, the absence of a correlation of relative power with the AEC-c does imply the presence of a robust effect.

\section{Collinearity between functional connectivity measures}

High correlations between the different functional connectivity measures have been found. This might not be surprising because, in principle, many were designed to explain similar phenomena. In our test setting, for example, high correlations were found between the imaginary coherence, PLI and wPLI. With $r$ values around 0.9 and higher, there is very high collinearity between these measures. Although the theoretical backgrounds and methods of calculation differ [21, 49,50], these measures yielded almost identical results when comparing $\mathrm{AD}$ versus $\mathrm{SCD}$. The AEC-c only showed fairly high correlations with its uncorrected counterpart (AEC).

\section{Disease severity and functional connectivity}

On average, the significant correlations between the level of functional connectivity and the MMSE score were weak. There are several potential explanations for this observation. The MMSE is a general test of cognition but emphasizes on memory function whilst $\mathrm{AD}$ patients also suffer from deficits in, for example, language or executive functioning. Other neuropsychological tests might have observed other effects. Furthermore, correlations were made between global levels of connectivity where regional connectivity could have stronger correlations with dysfunction in certain cognitive domains [23].

\section{Strengths, limitations and future directions}

The major strengths of our study were the large wellcharacterized sample size, a study design with internal validation, the use of the most recent NIA-AA AD research framework [13] with the availability of multiple biomarkers, rigorous correction for potential confounders and relative power, and the fact that multiple commonly used functional connectivity measures were tested. Previous studies do not only differ in the use of certain functional connectivity measures but also in epoch selection (no selection, visual selection or automatic selection), artefact rejection and other preprocessing steps. As there is no consensus on which method to use, we preferred to only do a protocolized visual selection by trained and experienced technicians based on our previous experience. To our knowledge, no other pre-processing methods have been proven to be superior in 21-channel EEG analyses. Potential limitations include the absence of an external validation cohort. Additionally, we have not investigated all available functional connectivity measures but made a selection based on commonly used measures and the previously reported intra-subject reliability of these measures [31]. It should also be considered that the reproducibility of functional connectivity in eyes-open EEG or task EEG data was not investigated. Moreover, additional research is needed to investigate the discriminative value of these reproducible measures between disease stages or the association with more specific tasks of different cognitive domains.

Future research, such as clinical trials or connectivity and network studies, could yield false positive or negative results by using unreliable functional connectivity measures or by using these measures in an invalid bandwidth. The results of this study provide information on 
which changes in functional connectivity are reproducible for Alzheimer's disease and to what extend they correlate with disease severity. This enables future studies to narrow-down and predefine functional connectivity measures as outcome measures.

\section{Conclusion}

In this large cross-sectional cohort study of wellcharacterized patients with AD and SCD, the AEC-c in the alpha and beta bands showed the most reproducible changes in functional connectivity, independent of various influencing factors, and strongest correlation with disease severity. Phase-based measures, with correction for volume conduction, observed strong effects in the theta band. These results might offer our research field some directions in solving the 'reproducibility crisis'.

\section{Supplementary information}

Supplementary information accompanies this paper at https://doi.org/10. 1186/s13195-020-00632-3.

Additional file 1: Supplementary Table 1. Difference in functional connectivity (FC) between SCD and AD subjects estimated by ANOVA model 1 (correction for age and gender). Supplementary Table 2. Difference in functional connectivity (FC) between SCD and AD subjects estimated by ANOVA model 2 (correction for age, gender and global relative power). Supplementary Figure S1. Topographical distribution of the median difference in Z-score of the PLI and AEC-c between the $S C D$ and $A D$ subjects. Supplementary Figure S2A \& 2B. Summary of observed differences in ANOVA model 1, shown as effect size (Cohen's d), between $A D$ and SCD subjects in different regions for the AEC-C and PLI in each bandwidth. Supplementary Figure S3A-C. Correlation coefficients ( $r$ ) between functional connectivity measures in the theta (A), alpha (B) and beta (C) bandwidths. In each figure, the coefficients on the right are corrected for changes in relative power and the coefficients on the left are the uncorrected values.

\section{Abbreviations}

AD: Alzheimer's disease; SCD: Subjective cognitive decline;

EEG: Electroencephalography; Coh: Coherence; iCoh: Imaginary coherence;

PLV: Phase locking value; AEC: Amplitude envelope correlation; AEC-C: AEC with leakage correction; PLI: Phase lag index; wPLI: Weighted PLI;

CSF: Cerebrospinal fluid; A $\beta$ : Amyloid beta; t-tau: Total tau; $p$ tau: Phosphorylated tau; MMSE: Mini-Mental State Exam; CNS: Central nervous system; MTA: Medial Temporal Atrophy score; PET: Positron emission tomography; MRI: Magnetic resonance imaging

\section{Acknowledgements}

We would like to thank all participants of the Amsterdam Dementia Cohort and employees of the VUmc Alzheimer Center Amsterdam for participating in the cohort and/or providing us with valuable information.

\section{Authors' contributions}

CTB, DNS, AAG and CJS were responsible for the study design. HdW, AAG and CJS were involved in the data acquisition. CTB performed the analysis and interpreted the data together with DNS, AAG and CJS. The manuscript was written by CTB and carefully revised by PhS, HdW, DNS, AAG and CJS. CTB is the corresponding author. The authors read and approved the final manuscript.

\section{Funding}

Not applicable.

\section{Availability of data and materials}

The datasets used and/or analysed during the current study are available from the corresponding author on reasonable request.

Ethics approval and consent to participate

The local medical ethics committee of VU University Medical Center has approved a general protocol for biobanking and using the clinical data for research purposes.

\section{Consent for publication}

Not applicable.

\section{Competing interests}

The authors declare that they have no competing interests.

Received: 28 October 2019 Accepted: 18 May 2020

Published online: 03 June 2020

\section{References}

1. Prince MJ. World Alzheimer report 2015: the global impact of dementia: an analysis of prevalence, incidence, cost and trends: Alzheimer's Disease International; 2015.

2. Jeong J. EEG dynamics in patients with Alzheimer's disease. Clin Neurophysiol. 2004;115(7):1490-505.

3. Dauwels J, Vialatte F, Cichocki A. Diagnosis of Alzheimer's disease from EEG signals: where are we standing? Curr Alzheimer Res. 2010;7(6):487-505.

4. van Straaten EC, Scheltens P, Gouw AA, Stam CJ. Eyes-closed task-free electroencephalography in clinical trials for Alzheimer's disease: an emerging method based upon brain dynamics. Alzheimers Res Ther. 2014; 6(9):86.

5. Engels MM, Stam CJ, van der Flier WM, Scheltens $P$, de Waal $H$, van Straaten EC. Declining functional connectivity and changing hub locations in Alzheimer's disease: an EEG study. BMC Neurol. 2015;15:145.

6. Stam CJ. Nonlinear dynamical analysis of EEG and MEG: review of an emerging field. Clin Neurophysiol. 2005;116(10):2266-301.

7. Boerman $\mathrm{R}$, Scheltens $\mathrm{P}$, Weinstein $\mathrm{H}$. Clinical neurophysiology in the diagnosis of Alzheimer's disease. Clin Neurol Neurosurg. 1994;96(2):111-8.

8. Welch $P$. The use of fast Fourier transform for the estimation of power spectra: a method based on time averaging over short, modified periodograms. IEEE Trans Audio Electroacoust. 1967;15(2):70-3.

9. Cassani R, Estarellas M, San-Martin R, Fraga FJ, Falk TH. Systematic review on resting-state EEG for Alzheimer's disease diagnosis and progression assessment. Dis Markers. 2018;2018:5174815.

10. de Haan W, van der Flier WM, Koene T, Smits LL, Scheltens P, Stam CJ. Disrupted modular brain dynamics reflect cognitive dysfunction in Alzheimer's disease. Neuroimage. 2012;59(4):3085-93.

11. Delbeuck $X$, Van der Linden M, Collette F. Alzheimer' disease as a disconnection syndrome? Neuropsychol Rev. 2003;13(2):79-92.

12. McKhann GM, Knopman DS, Chertkow H, Hyman BT, Jack CR Jr, Kawas CH, et al. The diagnosis of dementia due to Alzheimer's disease: recommendations from the National Institute on Aging-Alzheimer's Association workgroups on diagnostic guidelines for Alzheimer's disease. Alzheimers Dement. 2011;7(3):263-9.

13. Jack CR Jr, Bennett DA, Blennow K, Carrillo MC, Dunn B, Haeberlein SB, et al. NIA-AA research framework: toward a biological definition of Alzheimer's disease. Alzheimers Dement. 2018;14(4):535-62.

14. van Diessen E, Numan T, van Dellen E, van der Kooi AW, Boersma M, Hofman D, et al. Opportunities and methodological challenges in EEG and MEG resting state functional brain network research. Clin Neurophysiol. 2015;126(8):1468-81.

15. Babiloni C, Barry RJ, Basar E, Blinowska KJ, Cichocki A, Drinkenburg W, et al. International Federation of Clinical Neurophysiology (IFCN) - EEG research workgroup: recommendations on frequency and topographic analysis of resting state EEG rhythms. Part 1: applications in clinical research studies. Clin Neurophysiol. 2020;131(1):285-307.

16. Baker M. 1,500 scientists lift the lid on reproducibility. Nature. 2016; 533(7604):452-4.

17. Munafò MR, Nosek BA, Bishop DVM, Button KS, Chambers CD, Percie du Sert N, et al. A manifesto for reproducible science. Nat Human Behav. 2017; 1(1):0021. 
18. Locatelli T, Cursi M, Liberati D, Franceschi M, Comi G. EEG coherence in Alzheimer's disease. Electroencephalogr Clin Neurophysiol. 1998;106(3): 229-37.

19. Brunovsky M, Matousek M, Edman A, Cervena K, Krajca V. Objective assessment of the degree of dementia by means of EEG. Neuropsychobiology. 2003:48(1):19-26.

20. Adler G, Brassen S, Jajcevic A. EEG coherence in Alzheimer's dementia. J Neural Transm (Vienna). 2003;110(9):1051-8.

21. Stam CJ, Nolte G, Daffertshofer A. Phase lag index: assessment of functional connectivity from multi channel EEG and MEG with diminished bias from common sources. Hum Brain Mapp. 2007;28(11):1178-93.

22. Yu M, Gouw AA, Hillebrand A, Tijms BM, Stam CJ, van Straaten EC, et al. Different functional connectivity and network topology in behavioral variant of frontotemporal dementia and Alzheimer's disease: an EEG study. Neurobiol Aging. 2016:42:150-62.

23. Ranasinghe KG, Hinkley LB, Beagle AJ, Mizuiri D, Dowling AF, Honma SM, et al. Regional functional connectivity predicts distinct cognitive impairments in Alzheimer's disease spectrum. Neuroimage Clin. 2014;5:385-95.

24. Hsiao F-J, Wang Y-J, Yan S-H, Chen W-T, Lin Y-Y. Altered oscillation and synchronization of default-mode network activity in mild Alzheimer's disease compared to mild cognitive impairment: an electrophysiological study. PLoS One. 2013;8(7):e68792-e.

25. de Waal H, Stam CJ, Lansbergen MM, Wieggers RL, Kamphuis PJ, Scheltens $P$, et al. The effect of souvenaid on functional brain network organisation in patients with mild Alzheimer's disease: a randomised controlled study. PLoS One. 2014;9(1):e86558.

26. Dukic S, McMackin R, Buxo T, Fasano A, Chipika R, Pinto-Grau M, et al. Patterned functional network disruption in amyotrophic lateral sclerosis. Hum Brain Mapp. 2019;40(16):4827-42.

27. Schoonheim MM, Geurts JJ, Landi D, Douw L, van der Meer ML, Vrenken H, et al. Functional connectivity changes in multiple sclerosis patients: a graph analytical study of MEG resting state data. Hum Brain Mapp. 2013;34(1):52-61

28. Tewarie P, Steenwijk MD, Brookes MJ, Uitdehaag BMJ, Geurts JJG, Stam CJ, et al. Explaining the heterogeneity of functional connectivity findings in multiple sclerosis: an empirically informed modeling study. Hum Brain Mapp. 2018;39(6):2541-8

29. de Haan W, van Straaten ECW, Gouw AA, Stam CJ. Altering neuronal excitability to preserve network connectivity in a computational model of Alzheimer's disease. PLoS Comput Biol. 2017;13(9):e1005707.

30. Garces P, Martin-Buro MC, Maestu F. Quantifying the test-retest reliability of magnetoencephalography resting-state functional connectivity. Brain Connect. 2016:6(6):448-60.

31. Colclough GL, Woolrich MW, Tewarie PK, Brookes MJ, Quinn AJ, Smith SM. How reliable are MEG resting-state connectivity metrics? Neuroimage. 2016; 138:284-93.

32. Nunez PL, Srinivasan R, Westdorp AF, Wijesinghe RS, Tucker DM, Silberstein $\mathrm{RB}$, et al. EEG coherency. I: statistics, reference electrode, volume conduction, Laplacians, cortical imaging, and interpretation at multiple scales. Electroencephalogr Clin Neurophysiol. 1997;103(5):499-515.

33. Herreras O. Local field potentials: myths and misunderstandings. Front Neural Circuits. 2016;10:101

34. van der Flier WM, Scheltens P. Amsterdam dementia cohort: performing research to optimize care. J Alzheimers Dis. 2018;62(3):1091-111.

35. Scheltens P, Launer LJ, Barkhof F, Weinstein HC, van Gool WA. Visual assessment of medial temporal lobe atrophy on magnetic resonance imaging: interobserver reliability. J Neurol. 1995;242(9):557-60.

36. Tijms BM, Willemse EAJ, Zwan MD, Mulder SD, Visser PJ, van Berckel BNM, et al. Unbiased approach to counteract upward drift in cerebrospinal fluid amyloid-beta 1-42 analysis results. Clin Chem. 2018;64(3):576-85.

37. Frisoni GB, Bocchetta M, Chetelat G, Rabinovici GD, de Leon MJ, Kaye J, et al. Imaging markers for Alzheimer disease: which vs how. Neurology. 2013;81(5):487-500.

38. Wardlaw JM, Smith EE, Biessels GJ, Cordonnier C, Fazekas F, Frayne R, et al. Neuroimaging standards for research into small vessel disease and its contribution to ageing and neurodegeneration. Lancet Neurol. 2013;12(8): 822-38

39. Gouw AA, Alsema AM, Tijms BM, Borta A, Scheltens P, Stam CJ, et al. EEG spectral analysis as a putative early prognostic biomarker in nondemented amyloid positive subjects. Neurobiol Aging. 2017;57:133-42.

40. Stam CJ. "Brainwave" software version 0.9.152.12262019 [cited 20197 2019]. Available from: http://home.kpn.nl/stam7883/brainwave.html.
41. Babiloni C, Lizio R, Marzano N, Capotosto P, Soricelli A, Triggiani Al, et al. Brain neural synchronization and functional coupling in Alzheimer's disease as revealed by resting state EEG rhythms. Int J Psychophysiol. 2016;103:88-102.

42. Koelewijn L, Bompas A, Tales A, Brookes MJ, Muthukumaraswamy SD, Bayer A, et al. Alzheimer's disease disrupts alpha and beta-band resting-state oscillatory network connectivity. Clin Neurophysiol. 2017;128(11):2347-57.

43. Guevara MA, Corsi-Cabrera M. EEG coherence or EEG correlation? Int J Psychophysiol. 1996;23(3):145-53.

44. Lachaux JP, Rodriguez E, Martinerie J, Varela FJ. Measuring phase synchrony in brain signals. Hum Brain Mapp. 1999;8(4):194-208.

45. Mormann F, Lehnertz K, David P, Elger CE. Mean phase coherence as a measure for phase synchronization and its application to the EEG of epilepsy patients. Physica D Nonlinear Phenomena. 2000;144(3):358-69.

46. Bruns A. Fourier-, Hilbert- and wavelet-based signal analysis: are they really different approaches? J Neurosci Methods. 2004:137(2):321-32.

47. Bruns A, Eckhorn R, Jokeit $H$, Ebner A. Amplitude envelope correlation detects coupling among incoherent brain signals. Neuroreport. 2000;11(7):1509-14.

48. Hipp JF, Hawellek DJ, Corbetta M, Siegel M, Engel AK. Large-scale cortical correlation structure of spontaneous oscillatory activity. Nat Neurosci. 2012;15(6):884-90.

49. Nolte G, Bai O, Wheaton L, Mari Z, Vorbach S, Hallett M. Identifying true brain interaction from EEG data using the imaginary part of coherency. Clin Neurophysiol. 2004;115(10):2292-307.

50. Vinck M, Oostenveld R, van Wingerden M, Battaglia F, Pennartz CMA. An improved index of phase-synchronization for electrophysiological data in the presence of volume-conduction, noise and sample-size bias. Neurolmage. 2011:55(4):1548-65.

51. Conover WJ, Iman RL. Rank transformations as a bridge between parametric and nonparametric statistics. Am Stat. 1981;35(3):124-9.

52. Cohen J. Statistical power analysis for the behavioral sciences. Academic press; 2013 .

53. Benjamini $Y$, Hochberg $Y$. Controlling the false discovery rate - a practical and powerful approach to multiple testing. J R Stat Soc Ser B Statist Methodol. 1995;57(1):289-300.

54. Engel Andreas K, Gerloff C, Hilgetag Claus C, Nolte G. Intrinsic coupling modes: multiscale interactions in ongoing brain activity. Neuron. 2013;80(4):867-86.

55. Reinhart RMG, Nguyen JA. Working memory revived in older adults by synchronizing rhythmic brain circuits. Nat Neurosci. 2019;22(5):820-7.

56. Nunez P, Poza J, Gomez C, Rodriquez-Gonzalez V, Hillebrand A, Tola-Arribas $M A$, et al. Characterizing the fluctuations of dynamic resting-state electrophysiological functional connectivity: reduced neuronal coupling variability in mild cognitive impairment and dementia due to Alzheimer's disease. J Neural Eng. 2019;16(5):056030.

57. Ruiz-Gomez SJ, Hornero R, Poza J, Maturana-Candelas A, Pinto N, Gomez C. Computational modeling of the effects of EEG volume conduction on functional connectivity metrics. Application to Alzheimer's disease continuum. J Neural Eng. 2019;16(6):066019.

58. Perl DP. Neuropathology of Alzheimer's disease. Mt Sinai J Med. 2010;77(1):32-42.

59. McKeith IG, Boeve BF, Dickson DW, Halliday G, Taylor JP, Weintraub D, et al. Diagnosis and management of dementia with Lewy bodies: fourth consensus report of the DLB Consortium. Neurology. 2017;89(1):88-100.

60. van der Zande JJ, Gouw AA, van Steenoven I, Scheltens P, Stam CJ, Lemstra AW. EEG characteristics of dementia with Lewy bodies, Alzheimer's disease and mixed pathology. Front Aging Neurosci. 2018;10:190.

61. Guevara R, Velazquez JL, Nenadovic V, Wennberg R, Senjanovic G, Dominguez LG. Phase synchronization measurements using electroencephalographic recordings: what can we really say about neuronal synchrony? Neuroinformatics. 2005;3(4):301-14.

62. Winter WR, Nunez PL, Ding J, Srinivasan R. Comparison of the effect of volume conduction on EEG coherence with the effect of field spread on MEG coherence. Stat Med. 2007:26(21):3946-57.

63. Tewarie P, Hunt BAE, O'Neill GC, Byrne A, Aquino K, Bauer M, et al. Relationships between neuronal oscillatory amplitude and dynamic functional connectivity. Cereb Cortex. 2019;29(6):2668-81.

\section{Publisher's Note}

Springer Nature remains neutral with regard to jurisdictional claims in published maps and institutional affiliations. 